\title{
Circumstellar Envelopes of Peculiar and Normal J-Type Stars
}

\author{
S. LORENZ MARTINS \\ Observatorio Nacional-DAGE, Rio de Janeiro, Brazil
}

Late-type stars present circumstellar envelopes which contain gas and grains. In the case of carbon stars, stellar flux is absorbed by the envelope and re-emitted by carbon-rich grains in the infrared. Several compounds have been predicted to condense in these environments: graphite grains, amorphous carbon grains, silicon carbide $(\mathrm{SiC})$, and also $\mathrm{MgS}$ grains. J-type stars are identified in the visible range as carbon-rich and they can be separated into two distinct groups, according to their dust envelope properties: (1) normal J-type stars, which have carbon-rich envelopes, and (2) peculiar J-type stars, which have oxygen-rich envelopes. The modelling of normal and peculiar J-type envelopes has been performed by us using a Monte-Carlo type code for solving the radiative tranfer. For normal J-type stars two kinds of grains were considered simultaneously: amorphous carbon (A.C.), and silicon carbide (SiC). Silicate grains were used in modelling the peculiar ones. The temperatures of central stars and some characteristics of the circumstellar shell such as extinction opacities and its extension were determined by fitting the flux curves. The $\mathrm{SiC}$ /A.C. ratio as well as the energy distributions and temperature law have been studied. The results show that normal J-type carbon stars have thin envelopes (with extinction opacities about 0.02) and intermediate $\mathrm{SiC} /$ A.C. ratios. Peculiar J-type stars have thicker envelopes: their extinction opacities are about 0.60 . Based on these results, two alternative scenarios are discussed: (1) Normal J-type carbon stars may be an intermediate group between the Groups I and II introduced by Lorenz-Martins \& Lefêvre (1994, $A \mathcal{G} A, 291,831$ ). (2) J-type stars (normal and peculiar) could form an independent evolutionary sequence apart from that proposed for ordinary carbon stars.

This work was supported by CNPq-Brazil. 